\title{
RUN-TO-RUN CONTROL STRATEGY FOR DIABETES MANAGEMENT
}

\author{
F.J. Doyle III ${ }^{1}$, B. Srinivasan ${ }^{2}$, and D. Bonvin ${ }^{2}$ \\ ${ }^{1}$ Department of Chemical Engineering, University of Delaware, Newark DE, USA \\ ${ }^{2}$ Institut d'Automatique, EPFL, Lausanne, Switzerland \\ Email of corresponding author: fdoyle@udel.edu
}

\begin{abstract}
An approach to insulin injection therapy is introduced that draws from recent ideas in run-to-run control of batch chemical reactors. An analogy is drawn between repeated meal profiles for a diabetic patient and repeated operations of typical batch reactor recipes. The method relies on measurements of blood glucose and does not require a mathematical model. Results are shown for simulation case studies involving a detailed pharmacokinetic model.

Keywords - Diabetes control, glucose control, run-to-run control.
\end{abstract}

\section{Introduction}

Type I diabetes mellitus has received considerable attention in biomedical research, owing to the large fraction of the population which is affected by the disease (current estimate is 16 million worldwide). The primary manifestation of the disease is large fluctuations in the patient's blood glucose level. Short term implications of low glucose levels are quite serious (diabetic coma), and the longterm implications of varying glucose levels (nephropathy, retinopathy, and other tissue damage ) have received increasing attention in such forums as the Diabetes Control and Complications Trial (DCCT) [1], [2].

The approaches to glucose regulation are varied, with the conventional method consisting of 3-5 daily insulin injections with quantities of insulin based on 4-8 daily invasive glucose measurements. To date, this form of therapy has been unable to restore metabolism to a healthy patient state, and wide glucose fluctuations continue to occur for many patients. According to the DCCT, blood glucose should be controlled within the range of $60-120$ $\mathrm{mg} / \mathrm{dl}$. Research is underway for alternative methods, including beta cell transplants, insulin pumps, and oral or nasal delivery means. A survey of some of these approaches can be found in [3] and [4].

Control theoretic developments have focused primarily on the insulin pump regimens, with some of the early work dating to the BIOSTATOR ${ }^{T M}$ algorithm [5]. One of the early studies considered a hybrid formulation of an injection combined with continuous delivery [6]. However, the injection timing and amount were fixed in that analysis. A key issue in control studies is the source of the insulin injection, either intravenous or subcutaneous. A nice review of the two areas is provided in a pair of recent articles $([3],[4])$. Two key issues are the ease with which insulin can be administered in the respective sites, and the relatively longer time constant associated with the uptake of insulin delivered subcutaneously.

Another trend in the diabetes management area is the increasing availability of glucose measurement information for the patient. Recent developments include the AccuChek system from Roche Diagnostics and the GlucoWatch from Cygnus. Although these systems do not provide a continuous glucose reading, the frequency rates are approaching several samples per hour, which is an effective range for a system response that is on the order of several hours. There is an unprecedented amount of information available for the patients to use in the customization of their insulin therapy.

\section{Patient Protocol as a Batch-to-Batch Control Problem}

We propose in this paper a technique for optimizing a patient's insulin therapy (timing, amount) through the use of so-called run-to-run control [7]. This technique has found application in the chemical industry for the optimal operation of batch reactors which undergo repeated executions of a similar recipe.

The similarities between the diabetic patient protocol and the batch reactor recipe which motivate the application of this technique are:

- the recipe (24-hour cycle) for a human patient consists of a repeated meal protocol (typicall 3 meals) with some variance on meal type, timing, and duration,

- there is not an accurate dynamic model available to describe the detailed glucose response for an individual to the meal profile, and

- there are selected measurements available which might be used to characterize the "quality" of the response for a 24-hour day, including maximum and minimum glucose values.

As noted in the original reference [7], the key elements of the algorithm are that it is measurement-based (as opposed to model-based) and the independent variable of the control loop is the batch number. Thus, the solution is implemented as an open-loop policy for each batch (24-hour cycle), and the feedback allows refinement over successive batches (days).

Of particular interest in the present context is the fact that the limited measurement information of the patient's blood glucose level is translated into quality measurements ( $\max / \min$ glucose). In this way, the patient's sam- 
pling protocol does not need to be rigorously synchronized to a particular time every day, and the corresponding quality variables are exactly the type of variables that a medical professional would use to evaluate the efficiency of a particular insulin regimen.

\section{A. Description of the procedure}

The basic procedure from [7] is summarized below:

1. Parameterize the input profile for batch $k, u(t, k)$, as $\mathcal{U}(t, \nu(k))$ such that the input parameter vector, $\nu(k)$, and the controlled variable vector, $\psi(k)$, have the same dimension.

2. Choose an initial guess for $\nu(k), k=1$.

3 . Run the batch using the input $u(t, k)$ corresponding to $\nu(k)$. Compute $\psi(k)$ from the measurements, $y(t, k)$.

4. Update the input parameters using $\nu(k+1)=\nu(k)+$ $K\left(\psi^{r}-\psi(k)\right)$, with $K$ being an appropriate gain matrix and $\psi^{r}$ the reference values to be attained. Set $k=k+1$ and repeat steps 3-4 until convergence.

For the present application, $u(t, k)$ and $y(t, k)$ correspond to the insulin and glucose profiles, respectively. Two cases will be considered: a repeated single-meal and a three-meal 24-hour cycle. The running index $k$ represents the cycle number, i.e., the meal number in the first case and the day number in the second. The choice of $\nu(k)$ and $\psi(k)$ depends on the case considered and will be discussed next.

\section{B. Single meal study}

The two manipulated variables and the two controlled variables in this case are as follows:

- Manipulated variables: timing of insulin injection, $T$, and quantity of insulin injection, $Q$, i.e., $\nu^{T}=[T Q]$.

- Controlled variables: maximum value of glucose attained after the meal, $G_{\max }$, and minimum value of glucose attained after the peaking, $G_{\min }$, i.e., $\psi^{T}=$ $\left[G_{\max } G_{\min }\right]$. The maximum and minimum glucose concentrations are selected as convenient scalar measures of performance for a particular insulin regime.

In principle, a multivariable controller could be designed to maintain the two components of $\psi$ at their reference values $\psi^{r}$. However, the effects of the inputs are relatively decoupled for the case studies considered (as determined by the relative gain array (RGA)). The RGA analysis suggests a strategy which pairs the timing of insulin $T$ with maximum value of glucose $G_{\max }$, and the quantity of insulin $Q$ with minimum value of glucose $G_{\text {min }}$. So, the update laws for the timing and quantity of insulin injection are given below:

$$
\begin{aligned}
& \left.T(k+1)=T(k)+K_{T} \min \left(0,\left(G_{\max }^{r}-G_{\max }(k)\right)\right) 1\right) \\
& Q(k+1)=Q(k)+K_{Q} \max \left(0,\left(G_{\min }^{r}-G_{\min }(k)\right)(2)\right.
\end{aligned}
$$

Note that the controller used is of the integral type. To improve the rate of convergence, a proportional or a derivative term can be added, thus resulting in a PI or a PID controller, for the design of which standard controller design methodologies can be used.

A specific attribute of this problem is that any value of $G_{\max }(k)$ less than $G_{\max }^{r}$ is acceptable. So, no correction needs to be done when $\left(G_{\max }^{r}-G_{\max }(k)\right)$ is positive. A similar situation arises with $G_{\min }$ also, where no correction is necessary when $\left(G_{\text {min }}^{r}-G_{\min }(k)\right)$ is negative.

The important variables that need to be designed are the initial values $T(1)$ and $Q(1)$. In addition to $G_{\max }^{r}$ and $G_{\min }^{r}$ (desirable bounds on glucose), there exist hard limits $\bar{G}_{\max }$ and $\bar{G}_{\min }$ (absolute bounds that, if violated, lead to serious medical problems). The values $T(1)$ and $Q(1)$ should be so chosen that these hard constraints are satisfied for all cases. Also, the history of the patient can help choose initial values close enough to the eventual final values.

The gains $K_{T}$ and $K_{Q}$ are the next important parameters that have to be computed. Though the coupling is low, it might so happen that a fast decrease in $G_{\max }$ may cause an unacceptable dip in $G_{\text {min }}$ and vice-versa. Thus, the gains reflect a compromise between speed and accuracy.

\section{Extension to 24-hour cycle}

The previous study introduced the basic elements of the algorithm. One rather weak implicit assumption is that all meals appear similar. That assumption is relaxed here, where 3 meals are considered over a 24 -hour period as the basic cycle which is repeated.

In a normal day, there are three meals, each of which has fairly different caloric content. Thus the timing and the quantity of insulin used need to be different and adapted to the food habits. Thus, there are 6 manipulated variables ( $T$ and $Q$ for each meal) and 6 controlled variables $\left(G_{\max }\right.$ and $G_{\min }$ for each meal). This gives rise to a $6 \times 6$ multivariable control problem. However, as before, there is very strong decoupling between manipulated and controlled variables. Also, the morning meal and insulin intake have negligible influence on the glucose evolution during lunch and dinner. The mathematical details of the control development parallel the previous case, with the obvious increase in dimension, and are omitted in this paper. The application of this algorithm to a simulation patient model is reported in the next section.

\section{Simulated Patient Case Study}

The case study utilizes a detailed (19th order) compartmental model as a virtual patient to demonstrate the algorithm [9], [10]. The full model consists of 6 compartments to describe the metabolism of glucose and insulin in the body, with several compartments subdivided into separate tissue and capillary subspaces. The model is used to relate two inputs (meal ingestion, intravenous insulin injection) to the key output (blood glucose). The partic- 
ular parameter values and operating conditions employed in this simulation are detailed in [10].

As discussed earlier, the RGA calculated between the manipulated inputs (insulin timing/amount) and outputs ( $\max / \mathrm{min}$ glucose) is effectively diagonal:

$$
R G A_{\text {Sorensen }}=\left[\begin{array}{cc}
1.0126 & -0.0126 \\
-0.0126 & 1.0126
\end{array}\right]
$$

The reference values were $122 \mathrm{mg} / \mathrm{dl}$ for maximum glucose and $83.5 \mathrm{mg} / \mathrm{dl}$ for the minimum glucose (nominal patient has a basal glucose value of $87 \mathrm{mg} / \mathrm{dl}$ ). The hard constraints that were imposed on the glucose range were 207 and 72 , respectively.

The results of this case study are summarized in Figures 1 through 4 . The first figure depicts the final (optimal) insulin profile determined after 20 meals. The progressive improvement is depicted in the next 2 figures which show the maximum and minimum glucose values, respectively. It can be seen that the "optimal" maximum glucose is achieved after several meals, while the "optimal" minimum glucose level is achieved rather quickly. The fluctuations which appear in the profiles are the partially the result of a random signal (approximately 5 per cent relative standard deviation) employed for the actual meal size. Figure 4 shows the resulting glucose profile after the last iteration.

With regard to an actual patient trial, these results suggest that a relatively short trial period could be employed (2-3 days) after which the patient's new regimen could be established. In this manner, the proposed approach could be used either in a continuous fashion for constant finetuning, or on a periodic basis to allow "re-calibration".

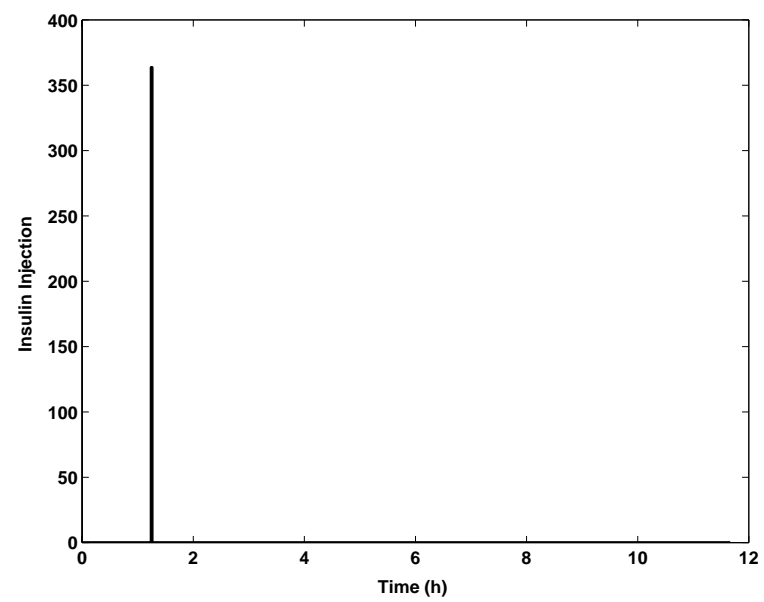

Fig. 1. Insulin injection profile calculated for a single meal response

\section{A. Extension to 24-hour cycle}

The same simulation model was utilized in the more practical setting of a 3 -meal (batch) cycle. The relative gain array supports a decentralized strategy which pairs

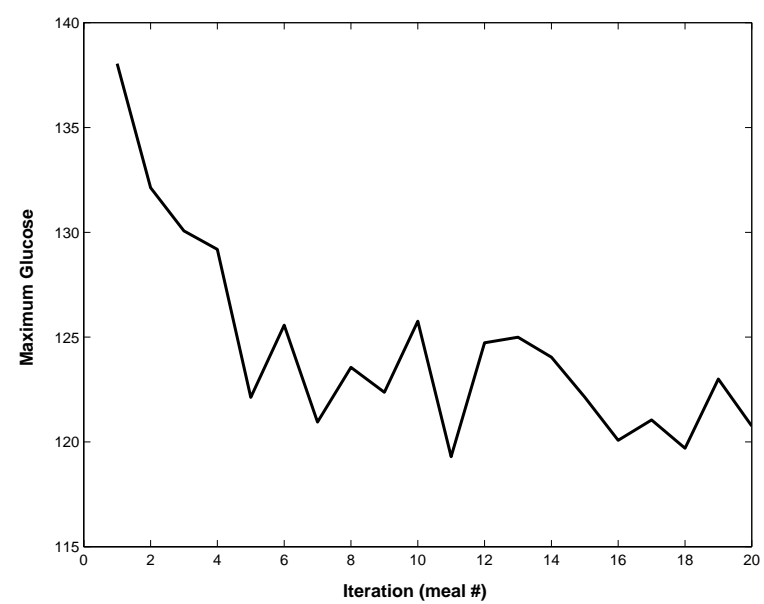

Fig. 2. Maximum glucose level versus iteration for a single meal response

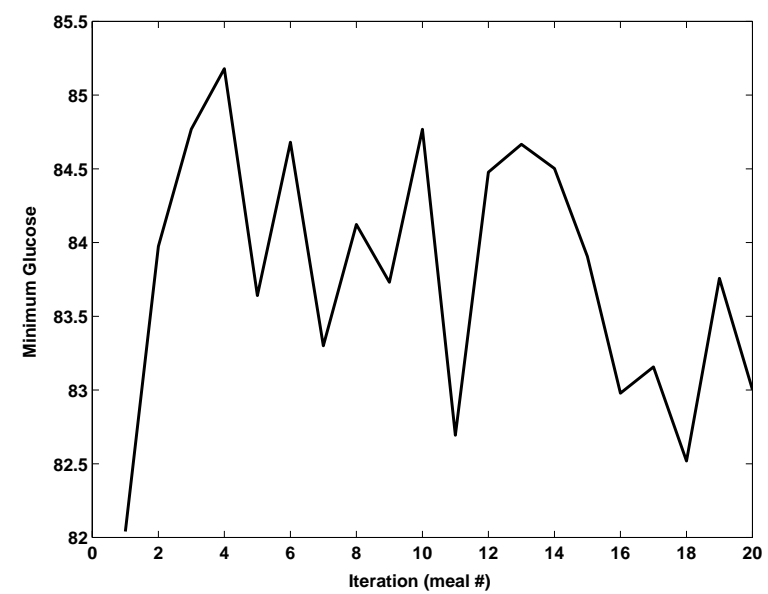

Fig. 3. Minimum glucose level versus iteration for a single meal response

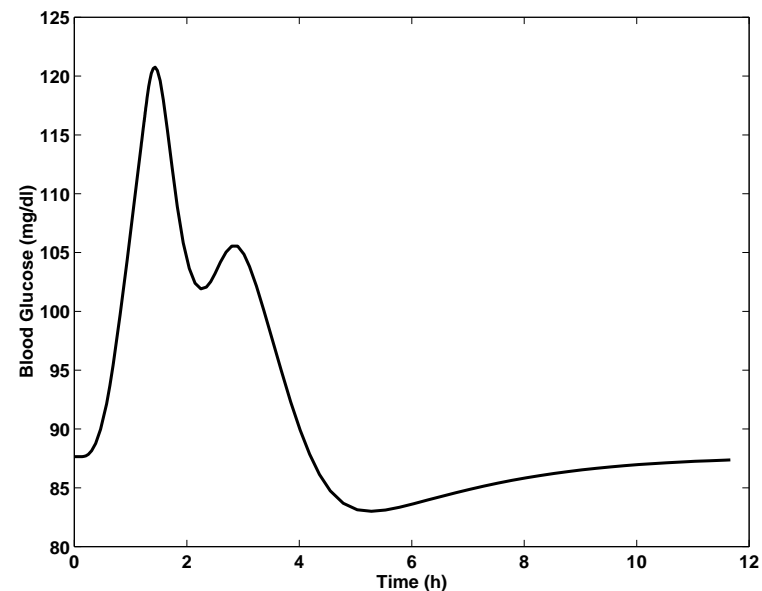

Fig. 4. Blood glucose profile calculated for a single meal response 
insulin dosage and timing with the corresponding minimum/maximum of the corresponding meal response. For the purposes of this study, the controller gains as the same for each meal - hence there are only two "tuning" knobs for this controller.

The results are summarized in Figures 5 and 6 . The final insulin profile after 10 cycles (days) is depicted in Figure 5. The corresponding optimal glucose profile is shown in Figure 6. It can be seen that the hypoglycemic excursions are quite small, and the hyperglycemic excursions are in an acceptable range.

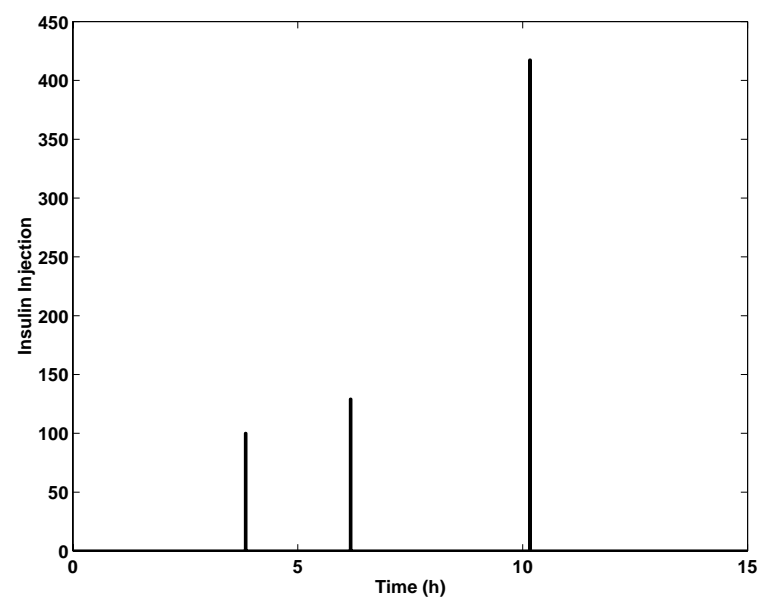

Fig. 5. Insulin injection profile calculated for a three meal response

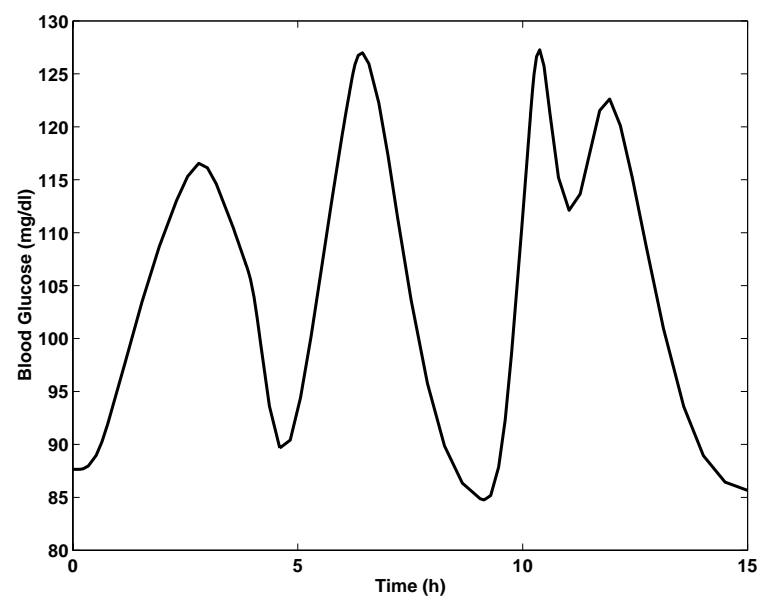

Fig. 6. Blood glucose profile calculated for a three meal response

\section{Conclusions and Extensions}

An approach to optimal insulin injection therapy for a diabetic patient is presented. The idea draws inspiration from batch-to-batch control strategies in the chemical process industry, and has been extended to the drug delivery context in this paper. The results show promise for both single meal and single day ( 3 meal) cycles. The con- vergence to an optimal insulin profile is typically accomplished in 5-10 cycles. Two of the particularly attractive features of the approach are: (i) there is no requirement for a mathematical model, and (ii) the measurement requirements are fairly modest (no high frequency, real-time measurements needed). The variables chosen for "quality" reflect the key medical attributes of the patient response (maximum and minimum glucose response).

The results could be generalized to a wide variety of drug delivery problems that contain some repetitive cycle of response to either a cyclic disturbance or a cyclic reference. The robustness of the approach to variations in the cycle (for example, meal timing and amount) is currently under investigation. An additional point for further investigation is the incorporation of a detailed description for subcutaneous transport of insulin in the patient model to more accurately reflect a standard insulin injection response.

\section{Acknowledgments}

The first author (FJD) would like to acknowledge the financial support of the EPFL for an extended summer (2000) visit during which the results in this paper were developed.

\section{REFERENCES}

[1] DCCT - The Diabetes Control and Complications Trial Research Group, "The effect of intensive treatment of diabetes on the development and progression of long-term complications in insulin-dependent diabetes mellitus," N. Engl. J. Med., vol. 329, pp. 977-986, 1993.

[2] DCCT - The Diabetes Control and Complications Trial Research Group, "The absence of a glycemic threshold for the development of long-term complications: The perspective of the diabetes control and complications trial," Diabetes, vol. 45, pp. 1289-1298, 1996.

[3] R.S. Parker, F.J. Doyle III, and N.A. Peppas, "The Intravenous Route to Blood Glucose Control," IEEE EMB Magazine, vol. 20, pp. 65-73, 2001.

[4] R. Bellazzi, G. Nucci, and C. Cobelli, "The Subcutaneous Route to Insulin-Dependent Diabetes Therapy," IEEE EMB Magazine, vol. 20, pp. 54-64, 2001.

[5] A.H. Clemens, "Feedback control dynamics for glucose controlled insulin infusion system," Med. Progr. Technol, vol. 6, pp. 91-98, 1979.

[6] M.E. Fisher, "A semiclosed-loop algorithm for the control of blood glucose levels in diabetics," IEEE Trans. Biomed. Eng., vol. 38, pp. 57-61, 1991.

[7] B. Srinivasan, C.J. Primus, D. Bonvin, and N.L. Ricker, "Run to Run Optimization via Constraint Control," in Proc. Int. Symp. Advanced Control in Chemical Processes, 2000, pp. 797802.

[8] R.N. Bergman, L.S. Phillips, and C. Cobelli, "Physiologic evaluation of factors controlling glucose tolerance in man," J. Clin. Invest., vol. 68, pp. 1456-1467, 1981.

[9] J.T. Sorensen, A Physiologic Model of Glucose Metabolism in Man and its Use to Design and Assess Improved Insulin Therapies for Diabetes, Ph.D. thesis, Department of Chemical Engineering, MIT, 1985.

[10] R. S. Parker, F. J. Doyle III, and N. A. Peppas, "A model-based algorithm for blood glucose control in type I diabetic patients," IEEE Trans. Biomed. Eng., vol. 46, no. 2, pp. 148-157, 1999. 